Relations industrielles

Industrial Relations

\title{
Les rivalités syndicales au Québec
}

\section{Jean-Réal Cadrin}

Volume 19, numéro 4, 1964

URI : https://id.erudit.org/iderudit/027522ar

DOI : https://doi.org/10.7202/027522ar

Aller au sommaire du numéro

\section{Éditeur(s)}

Département des relations industrielles de l'Université Laval

\section{ISSN}

0034-379X (imprimé)

1703-8138 (numérique)

Découvrir la revue

Citer cet article

Cadrin, J.-R. (1964). Les rivalités syndicales au Québec. Relations industrielles / Industrial Relations, 19(4), 502-504. https://doi.org/10.7202/027522ar

Tous droits réservés (C) Département des relations industrielles de l'Université Laval, 1964
Ce document est protégé par la loi sur le droit d'auteur. L’utilisation des services d'Érudit (y compris la reproduction) est assujettie à sa politique d'utilisation que vous pouvez consulter en ligne.

https://apropos.erudit.org/fr/usagers/politique-dutilisation/ 


\section{COMMENTAIRES}

Les rivalités syndicales au Québec

\section{Jean-Réat Cardin}

Il semble que le mouvement syndical au Québec soit à l'orée d'une certaine transformation aussi bien sur le plan des structures que sur celui des fonctions et des attitudes. Il a acquis graduellement depuis une quinzaine d'années, en dépit des hauts et des bas qui ont caractérisé cette période sur le plan des effectifs et de l'influence politique, une stature institutionnelle qui s'affermit chaque jour davantage et le fait peser d'un poids plus lourd dans la balance des intérêts communautaires.

Il n'est pas question ici d'attribuer au mouvement syndical québécois seul les raisons de cet état de choses; les transformations industrielles, les poussées du progrès technique dans certains secteurs, les nouvelles attitudes gouvernementales en matière d'éducation, de sécurité sociale et de législation du travail, les impératifs d'une économie en voie de «concertation», enfin, le climat de revendication sociale longtemps contenue mais déclenchée presque du jour au lendemain chez plusieurs groupes traditionnellement indifférents au syndicalisme, sont autant de causes, à notre avis, qui ont servi puissamment les intérêts du travail organisé dans les dernières années.

Il n'en reste pas moins que de larges avenues de progrès semblent s'ouvrir pour le syndicalisme au Québec, contrairement à ce qui s'offrait à lui il y a à peine quelques années. Il n'en tient donc qu'à lui de profiter de cette conjoncture redressée en utilisant au maximum les possibilités nouvelles qui se font jour actuellement.

Il n'est donc pas surprenant, si l'on aborde le problème en tenant compte des remarques que nous venons de faire, qu'une concurrence inter-syndicale accrue s'exprime ouvertement depuis quelque temps entre les syndicats affiliés à l'une ou l'autre des deux grandes centrales syndicales québécoises, la Confédération des Syndicats nationaux (C.S.N.) et la Fédération des Travailleurs du Québec (F.T.Q.).

Il n'y a pas à se scandaliser, loin de là, si l'on voit les syndicats membres de l'une et de l'autre, se faire la lutte afin de pouvoir organiser et représenter les salariés de différents secteurs nouvellement ouverts au syndicalisme ou du moins offrant de plus grandes possibilités en matière de reconnaissance syndicale et de négociations collectives. 
Tout ceci se situe strictement à l'intérieur des règles du jeu du syndicalisme libre là où existe la pluralité syndicale au niveau des centrales, c'est-à-dire là où des syndicats d'allégeance différente se font face, comme c'est le cas au Québec avec la présence de la C.S.N. d'une part, et le C.T.C. (Congrès du Travail du Canada) représenté sur le plan québécois par la F.T.Q. d'autre part.

En effet, pour simplifier l'analyse (car d'autres organisations existent, mais de beaucoup moindre importance) le Québec est la seule province canadienne, à toute fin pratique, où n'existe pas l'unité structurelle au sommet, au sein du C.T.C., ce dernier ayant à partager le pouvoir syndical avec la C.S.N., mouvement d'origine autochtone, bien intégré au milieu et affichant un dynamisme renouvelé et une volonté d'expansion dont les exemples restent rares dans l'histoire syndicale canadienne.

Tant et aussi longtemps que la lutte entre syndicats de différentes allégeances s'organise autour de nouveaux secteurs à occuper syndicalement, elle constitue, à vrai dire l'indice certain d'une saine vitalité chez les organismes en présence et la garantie probable que les travailleurs trouveront leur compte sous forme de services syndicaux répondant davantage à leurs besoins personnels.

Les grandes périodes d'expansion syndicale, en Amérique du Nord, et même en Europe, où pourtant les données du problème ne sont pas les mêmes, se sont faites la plupart du temps, à travers une rivalité syndicale qui, tout en portant en elle-même certains germes de faiblesse sur le plan des organisations en cause, n'en ont pas moins, en longue période, affermi l'institution syndicale dans son ensemble et permis aux individus membres des choix plus conformes à leurs besoins propres.

Là où l'on peut commencer à se poser des questions sérieuses au sujet des récentes luttes opposant syndicats de la C.S.N. à ceux du C.T.C. représentés au Québec par la F.T.Q., c'est lorsque ces conflits portent, non plus sur des groupes d'employés jusqu'ici non syndiqués, mais s'expriment à l'endroit de travailleurs faisant déjà partie d'un syndicat affilié à un titre ou à un autre à l'une des deux centrales.

Dans ce dernier cas, l'interprétation à donner de ces événements doit nécessairement être nuancée. Tout un traité de sociologie syndicale ne serait pas de trop pour analyser l'ensemble d'un tel problème d'une façon quelque peu satisfaisante. Disons d'abord seulement que l'élément de surprise qui s'attache forcément aux nouvelles faisant état de ces tensions présentes dans notre monde syndical, vient à notre avis du fait que le syndicalisme nord-américain, qui est aussi le nôtre, ne l'oublions pas, en est un de monopole de représentation, au niveau local, grâce à la procédure de l'accréditation du syndicat majoritaire. 
Deux syndicats concurrents ne peuvent, à toute fin pratique, coexister et représenter concurremment un même groupe d'employés.

De plus, étant donné les pactes de non-agression, les clauses de sécurité syndicale et les ouvertures trè réduites que la loi ménage aux salariés non satisfaits de leur organisation leur permettant de changer d'allégeance syndicale, on n'est pas habitué chez-nous à voir ces derniers évoluer librement d'un syndicat à un autre.

Ce régime, quoiqu'assurant une très grande stabilité institutionnelle au syndicalisme, n'en comporte pas moins des dangers réels pour les individus membres dont la liberté de choix est réduite presqu'à néant, en régime d'unité syndicale parfaite.

Si la concurrence entre les deux centrales québécoises a pour objet d'offrir un élément de choix et une possibilité de changement d'adhésion à des groupes de travailleurs qui le désirent, elle constitue un élément essentiel de la liberté syndicale et ne saurait faire l'objet d'aucune critique, loin de là. La sollicitation des travailleurs, faite par des moyens honnêtes et sans éléments de surenchère dans les tactiques utilisées, par chacune des centrales prétendant offrir quelque chose de mieux sur le plan idéologique, technique ou matériel, n'est qu'une chose bonne en soi.

$\mathrm{Si}$, au contraire, le syndicalisme se perd dans des épreuves de force stériles, dans un «maraudage » intempestif et non justifié, dans un pur but d'expansion matérielle et de prestige, ou pour satisfaire d'obscures visées individuelles de conquête idéologique non avouée, ses fins essentielles sont alors perverties car on ne recherche plus le bien des travailleurs, ni de l'industrie de laquelle ils vivent, en les faisant les jouets d'une institution dont ils sont, après tout la seule raison d'être.

Dans cette dernière hypothèse, le danger très grave, à notre avis, serait que l'«image» du monde syndical au Québec en sorte ternie aux yeux de l'opinion publique. Ce danger serait d'autant plus grand à mesure que les querelles tendent à se transposer au niveau des centrales mêmes car c'est alors le monde syndical tout entier, dans ses institutions les plus représentatives, qui en est atteint, et par ricochet inévitable les travailleurs qui en font partie.

Comme le préconisait M. Jean Marchand au dernier congrès de la C.S.N. à Québec, il serait urgent qu'un code d'éthique, ayant comme critère ultime le bien des salariés eux-mêmes, soit développé et mis au point afin qu'une concurrence syndicale néfaste ne fasse obstruction à la création éventuelle d'un front syndical uni, face aux autres partenaires sociaux, lorsqu'il s'agira pour le monde organisé du travail d'assumer sa part de responsabilités sur l'échiquier des décisions communautaires en matière économique et sociale. 